\title{
Evaluating Educational Intervention to Improve Drug Administration Practice in Out-Patient Children in Northwestern Nigeria
}

\author{
Chedi BAZ ${ }^{1 *}$, Abdu-Aguye $\mathrm{I}^{2}$ and Kwanashie $\mathrm{HO}^{2}$
}

${ }^{1}$ Department of Pharmacology, Bayero University Kano, Nigeria

${ }^{2}$ Department of Pharmacology and Therapeutics, Ahmadu Bello University, Zaria, Nigeria

\begin{abstract}
Drug administration practice of mothers from 20 facilities (12 primary and 8 secondary) in northwestern Nigeria were assessed prospectively with the aim of identifying the type, frequency and potential clinical significance of drug administration errors in paediatric outpatients. The data was analyzed and errors classified according to National Coordinating Council for Medication Error Reporting and Prevention taxonomy. Educational interventions were designed and administered to mothers of 10 (6 primary and 4 secondary) least performing facilities, while the remaining 10 facilities acted as control. The percentage of the parents that claimed to knew the correct dosage were relatively high (78\% to $93 \%)$ and differ significantly between the secondary and primary facilities. Further assessment shows that the dose of the drugs dispensed, the time/frequency of administration, and the duration of use were not known by $68.2 \%$ (330/484), 63.0\% (305/484), and $12.0 \%(58 / 484)$ of the mothers respectively. The overall total possibility of the drug administration errors significantly reduced in the right direction $(p<0.0005 ; d=4.27)$ after the intervention.
\end{abstract}

Keywords: Drug administration errors; Paediatric; Outpatient; Health facilities; Nigeria

\section{Introduction}

A drug administration error was defined as any discrepancy between printed or handwritten prescribers' orders and drug delivery to the patient, or deviation from a prescriber's valid prescription, official pharmacopeia or manufacturer's literature. Administration errors were classified into wrong timing; that is greater than one hour difference compared with the ordered time [1], wrong dose (quantity), wrong drug, wrong frequency, wrong duration and wrong route. Many drugs used in paediatric units are either unlicensed or used off-label [2]. The need to prepare dilutions or to open capsules may increase the risk of drug administration errors [3]. When computing drug doses, health care personnel may make mistakes, which may be life threatening [4]. In paediatric units, the physiological immaturity and widely variable body weight of the patients may increase the risk and impact of errors. The incidence of medication errors and the risk of serious errors occurring in children are significantly greater than in adults. In USA alone, it was estimated that 100-150 deaths occur annually in children due to medication errors [5]. Studies by Freyn [6], King [7] and Miller [8] showed the rate of medication errors in paediatric practice as follows: $3-37 \%$ prescribing, $5-58 \%$ dispensing and $72-75 \%$ administration errors. The household administration of liquid dosage form has been identified as one of the important factors contributing to medication error in paediatric patients [9]. Teaspoons used at home vary greatly in size, causing many children to be underdosed [10]. Since about $72 \%$ of the drugs were prescribed as syrups, this is an area of major public health concern [11].

Despite the awareness that paediatric patients are at increased risk for medication errors [12], little is known about their drug utilization and epidemiology of these errors. Much of the studies on drug utilization focused on general outpatient, few have been conducted in the paediatric setting and even fewer have concentrated on medication errors [13]. With our understanding of the problems and solutions of patient safety growing daily, it has become clear that the prescription, dispensing and administration of medication represent a substantial portion of preventable medical errors that occur in paediatric. The objective of this study was to identify the type, frequency and potential clinical significance of drug administration errors in paediatric outpatients with a view to intervening.

\section{Materials and Method}

\section{Study design}

The study was a cross sectional prospective survey involving twenty public health care institutions selected from Kano State by stratified random sampling using senatorial district as stratum. In each senatorial district, two secondary and four primary public health facilities were selected by simple random sampling using balloting system. Also the only available paediatric specialist and teaching hospitals were added to make-up the twenty facilities. After sampling, ten (four secondary and six primary) facilities were purposely selected to receive the intervention while the remaining acted as control.

\section{Inclusion and exclusion criteria}

The subjects included in this study were mothers that brought patients of either sex, aged 11 years or below with general illness. Mothers that present patients to the health care facilities for follow-up of chronic diseases or to receive services such as vaccination, and other specialized care services were excluded from the study sample.

\section{Data collection}

Consents of mothers that brought children to the selected facilities

*Corresponding author: Chedi BAZ, Department of Pharmacology, Bayero University Kano, Nigeria Tel: +234 8035970540; E-mail: b2chedi@yahoo.com

Received August 04, 2014; Accepted October 06, 2014; Published October 08 2014

Citation: Chedi BAZ, Abdu-Aguye I, Kwanashie HO (2014) Evaluating Educational Intervention to Improve Drug Administration Practice in Out-Patient Children in Northwestern Nigeria. Adv Pharmacoepidemiol Drug Saf 3: 162. doi:10.4172/2167 1052.1000162

Copyright: (C) 2014 Chedi BAZ, et al. This is an open-access article distributed under the terms of the Creative Commons Attribution License, which permits unrestricted use, distribution, and reproduction in any medium, provided the original author and source are credited. 
were sought at the point of exit. Those that accepted were recruited into the study and were asked whether they knew how to administer the drugs dispensed to their wards or not, the ones that claimed to know the correct dose were enlisted while the remaining were excluded. The data was collected in two waves:

(a) Pre-intervention (Baseline): Each mother was requested to (1) identify the drugs dispensed to her child from mix of other drugs, (2) state the route administration of each drug, (3) mentioned the times and number of days that each drug will be given and (4) mothers were provided with paracetamol syrup and all other requirements including a medium-sized teaspoon, at no cost. They were requested to measure one dose $(5 \mathrm{ml})$ three times the way they would normally do at home. After each pour, the quantity was withdrawn and measured using a syringe, the average was recorded. For the purpose of this study, failure to identify the right drugs, state right route, right duration, right time (schedule time \pm one hour) and measure right q Nigeria uantity $4.75 \mathrm{ml}$ to $5.25 \mathrm{ml}(5 \mathrm{ml} \pm 5 \%)$ were considered as potential administration errors. The errors were calculated as percentages and classified according to standardized taxonomy of classifying errors [14].

For the purpose of this study, request for clarification or expression of doubt before any aspect of the drug administration practice was considered as category A error, while an error that was intercepted during the administration practice was categorized as B error. Errors that involved non-antimicrobial drugs and/or drugs with wide therapeutic range were considered as category $\mathrm{C}$ error and where an antimicrobial drug or drug with narrow therapeutic index was involved it was categorized as D error. When several errors were made during the same administration were counted as one and the highest level of severity that applies during the course of the event was selected as the outcome category.

(b) Post-intervention: mothers were allowed to rest for 30 minutes during which the pre-intervention (baseline) data was analyzed; areas that need intervention identified and appropriate interventions administered. The baseline procedure was repeated prior to the intervention in the control facilities and 15 minutes after the intervention in the intervention facilities.

(c) Intervention Description: Mothers were trained on good drug administration skills and enlightened about consequences of medication errors and importance of adhering to dosage regimens. They were showed what a $5 \mathrm{~mL}$ dose looked like and the meanings of twice daily, thrice daily etc in terms of hours.

\section{(iv) Statistical Analysis}

The percentage error ( $\mathrm{pE}$ ) was calculated as the number of administrations with one or more errors $(\mathrm{nE})$ divided by the total number of observed drug administration (nA) times 100. Data collected was entered into HP laptop and coded. Analysis was carried out using Microsoft excel 2007 and SPSS version 15; values were express as mean \pm SEM. Drug administration errors were computed and compared between the primary and secondary facilities by Student's t-test. To evaluated the impact of the intervention, the difference (net gain scores) was computed by subtracting the mean difference (gain scores) of the control group (post minus pre) from mean difference (gain scores) of the intervention group as suggested by Tumwikirize et al. [15]. Plus sign (+) indicates increase and negative sign (-) signifies decrease. Secondly, mean differences of control and intervention groups were compared using unpaired Student's t-test $[16,17]$ and values of $p<0.05$ were considered statistically significant. Thirdly, to know if an observed difference is not only statistically significant but also important or meaningful, effect size [18] was calculated for each indicator. Cohen's effect size (d) was determined by mean difference of intervention group (m1) minus mean difference of control (m2)/pooled standard deviation. By convention the subtraction $(\mathrm{m} 1-\mathrm{m} 2)$, was done so that the difference is positive if it is in the direction of improvement or in the predicted direction and negative if in the direction of deterioration or opposite to the predicted direction. Cohen's $\mathrm{d}<0.2=$ trivial effect; $0.2-0.4=$ small effect; $0.5-0.8=$ moderate effect; $>0.8=$ large difference effect.

\section{Result}

During the study, 484 drug administration practices by 242 mothers were assessed. The percentage of the mothers that claimed to knew the correct dosage were relatively high (78\% to 93\%) and differ significantly between the secondary and primary facilities (Table 1), but this does not necessarily reflect reality because the response "Yes or I know the dose" was accepted as positive answer.

Further assessment of knowledge of those mothers that claimed to know the dose of drugs dispensed in relation to the quantity to administer, the time/frequency of administration, and the duration of use were not known by $68.2 \%$ (330/484), $63.0 \%$ (305/484), and $12.0 \%$ $(58 / 484)$ of the mothers respectively (Table 2$)$.

These errors occurred for more than $90 \%$ pre-intervention ally but significantly reduced in the right direction $(\mathrm{p}<0.0005 ; \mathrm{d}=4.27)$ after the intervention (Table 3).

\section{Discussions}

About $90 \%$ and $80 \%$ of parents in the secondary and primary health care facilities respectively claimed to know the correct dosage schedule, and significant $(\mathrm{p}<0.05 ; \mathrm{d}=1.22)$ increase was recorded in secondary facilities after the intervention. These figures, though higher than $55 \%$ to $68.3 \%$ reported in Bangladesh [19], Burkina Faso [20], Cambodia [21] and Indian [22], did not necessarily reflect reality since the response "Yes, I know the dose" was accepted as positive answer. The claim was proved wrong after the drug administration error analysis.

Assessment of possibilities of drug administration errors proved most parents that claimed to have adequate knowledge on dosage schedule wrong. This was evidenced by the high value of $88.5-100 \%$ total errors obtained in the both secondary and primary facilities before

Table 1: Percentage of parents who claimed to have adequate knowledge.

\begin{tabular}{|c|c|c|c|c|c|c|}
\hline & \multicolumn{2}{|c|}{ Control group } & \multicolumn{2}{|c|}{ Intervention group } & \multicolumn{2}{|c|}{ Group comparison } \\
\hline & Pre & Post & Pre & Post & Diff & P Value \\
\hline Sec. $(n=4)$ & $93.18 \pm 2.80$ & $92.65 \pm 2.70$ & $85.35 \pm 1.46$ & $89.75 \pm 1.11$ & 4.93 \\
\hline Pri. $(n=6)$ & $78.12 \pm 1.183$ & $80.85 \pm 1.842$ & $79.95 \pm 2.750$ & $84.48 \pm 1.721$ & 1.80 \\
\hline Overall $(\mathbf{n = 1 0})$ & $\mathbf{8 4 . 1 4} \pm \mathbf{2 . 7 5}$ & $\mathbf{8 5 . 5 7} \pm \mathbf{2 . 4 1}$ & $\mathbf{8 2 . 1 1} \pm \mathbf{1 . 8 9}$ & $\mathbf{8 6 . 5 9} \pm \mathbf{1 . 3 7}$ & $\mathbf{1 . 4 3}$ & $0.44 \mathrm{~ns}$ \\
\hline
\end{tabular}

Group comparison: Difference $(-)=$ decrease and $(+)=$ increase; $P$ value-ns-not significant; $a=<0.05 ; b=<0.005 ; c=<0.0005 ;$ Effect size $\left(\right.$ Cohen's d)- ${ }^{*}=0.2-0.4($ small);

${ }^{* *}=0.5-0.8$ (medium); ${ }^{* * *}=>0.8$ (strong); $(+)=$ improvement, $(-)=$ deterioration;

Facilities comparison $P$ value $-0=$ not significant; $1=<0.05 ; 2=<0.005 ; 3=<0.0005$ 


\begin{tabular}{|c|c|c|c|c|c|}
\hline Error Category & Type of Error & $\begin{array}{l}\text { Frequency of Errors } \\
\text { (n) }\end{array}$ & $\begin{array}{c}\text { Percentage } \\
(\%)\end{array}$ & $\begin{array}{l}\text { Pre-intervention } \\
\quad(n=242)\end{array}$ & $\begin{array}{l}\text { Post-intervention } \\
\quad(n=242)\end{array}$ \\
\hline & & & & Rate per 100 Parents & Rate per 100 Parents \\
\hline \multirow{3}{*}{$\begin{array}{c}\text { A } \\
\text { (Circumstances or events that have the capacity } \\
\text { to cause error) }\end{array}$} & TOTAL & 63 & 100.0 & 14.8 & 22.4 \\
\hline & Look Alike & 23 & 36.5 & 5.1 & 4.4 \\
\hline & $\begin{array}{l}\text { Inadequate } \\
\text { Information }\end{array}$ & 45 & 71.4 & 9.8 & 8.8 \\
\hline \multirow{4}{*}{$\begin{array}{c}\text { C } \\
\text { (An error occurred that reached the patient, but } \\
\text { may not cause patient harm) }\end{array}$} & TOTAL & 226 & 100.0 & 50.0 & 43.0 \\
\hline & Wrong Quantity & 214 & 94.7 & 48.8 & 39.6 \\
\hline & Wrong Time & 197 & 87.2 & 40.8 & 40.6 \\
\hline & Wrong Duration & 31 & 13.7 & 7.0 & 5.8 \\
\hline \multirow{4}{*}{$\begin{array}{l}\text { D } \\
\text { (An error occurred that required monitoring or } \\
\text { intervention to preclude harm) }\end{array}$} & TOTAL & 128 & 100.0 & 25.8 & 27.2 \\
\hline & Wrong Quantity & 116 & 90.6 & 24.2 & 23.7 \\
\hline & Wrong Time & 108 & 84.4 & 22.8 & 21.9 \\
\hline & Wrong Duration & 27 & 21.1 & 6.3 & 4.8 \\
\hline
\end{tabular}

Table 3: Percentages of Administration Error.

\begin{tabular}{|c|c|c|c|c|c|c|c|c|}
\hline & & \multicolumn{2}{|c|}{ Control group } & \multicolumn{2}{|c|}{ Intervention group } & \multicolumn{3}{|c|}{ Group comparison } \\
\hline & & Pre & Post & Pre & Post & Diff & P Value & Effect Size \\
\hline \multirow{3}{*}{$\begin{array}{l}\text { Category A (Circumstances or events that have the } \\
\text { capacity to cause error). }\end{array}$} & $\operatorname{Sec}(n=4)$ & $11.28 \pm 4.13$ & $3.85 \pm 3.85$ & $13.80 \pm 0.23$ & $1.68 \pm 1.68$ & -4.70 & $0.08^{\text {ns }}$ & $3.54^{* * *}$ \\
\hline & Pri $(n=6)$ & $21.83 \pm 1.30^{1}$ & $18.13 \pm 2.58^{1}$ & $21.13 \pm 1.52^{2}$ & $2.78 \pm 2.59^{n s}$ & -11.65 & $0.02^{\mathrm{a}}$ & $6.17^{\star \star \star}$ \\
\hline & $\begin{array}{l}\text { Overall } \\
(n=10)\end{array}$ & $17.61 \pm 2.41$ & $12.42 \pm 3.10$ & $18.20 \pm 1.49$ & $4.14 \pm 1.75$ & -8.87 & $0.0005^{c}$ & $4.07^{* * *}$ \\
\hline \multirow{3}{*}{$\begin{array}{l}\text { Category C (An error occurred that reached the } \\
\text { patient, but may not cause patient harm). }\end{array}$} & $\operatorname{Sec}(n=4)$ & $53.63 \pm 5.13$ & $54.55 \pm 3.36$ & $51.80 \pm 1.04$ & $34.60 \pm 0.64$ & -18.13 & $0.001^{\mathrm{b}}$ & $10.78^{* * *}$ \\
\hline & Pri $(n=6)$ & $49.52 \pm 2.08^{0}$ & $45.77 \pm 3.06^{0}$ & $47.42 \pm 2.63^{0}$ & $37.58 \pm 3.26^{0}$ & -6.08 & $0.07^{\text {ns }}$ & $2.32^{* * *}$ \\
\hline & $\begin{array}{l}\text { Overall } \\
(n=10)\end{array}$ & $51.16 \pm 2.32$ & $49.28 \pm 2.58$ & $36.39 \pm 1.96$ & $36.39 \pm 1.96$ & -10.90 & $0.002^{b}$ & $4.20^{* * *}$ \\
\hline \multirow{3}{*}{$\begin{array}{l}\text { Category } \mathbf{D} \text { (An error occurred that reached the } \\
\text { patient and required monitoring or intervention to } \\
\text { preclude harm). }\end{array}$} & $\operatorname{Sec}(n=4)$ & $23.63 \pm 5.05$ & $23.88 \pm 5.56$ & $34.35 \pm 0.78$ & $22.23 \pm 2.98$ & -12.30 & $0.02^{\mathrm{a}}$ & $5.51^{* \star *}$ \\
\hline & Pri $(n=6)$ & $28.60 \pm 1.68^{0}$ & $25.55 \pm 2.77^{0}$ & $31.45 \pm 1.90^{\circ}$ & $21.62 \pm 4.73^{\circ}$ & -6.78 & $0.03^{a}$ & $2.71^{* \star *}$ \\
\hline & $\begin{array}{l}\text { Overall } \\
(n=10)\end{array}$ & $26.61 \pm 2.24$ & $24.88 \pm 2.60$ & $32.61 \pm 1.23$ & $21.89 \pm 2.94$ & -8.99 & $0.005^{b}$ & $3.63^{* * *}$ \\
\hline \multirow[t]{3}{*}{ Total } & $\operatorname{Sec}(n=4)$ & $88.53 \pm 4.37$ & $82.28 \pm 6.69$ & $99.95 \pm 0.03$ & $58.58 \pm 3.55$ & -35.13 & $<0.0005^{c}$ & $15.46^{* * *}$ \\
\hline & Pri $(n=6)$ & $99.95 \pm 0.02^{1}$ & $89.45 \pm 2.64^{0}$ & $100.00 \pm 0.04^{0}$ & $64.95 \pm 2.83^{0}$ & -24.55 & $<0.0005^{c}$ & $16.92^{\star \star \star}$ \\
\hline & $\begin{array}{l}\text { Overall } \\
(n=10)\end{array}$ & $95.38 \pm 2.46$ & $86.58 \pm 3.11$ & $99.98 \pm 0.02$ & $62.40 \quad 2.33$ & -28.78 & $<0.0005^{c}$ & $11.17^{\star \star \star}$ \\
\hline
\end{tabular}

Group comparison: Difference $(-)=$ decrease and $(+)=$ increase; $P$ value-ns-not significant; $a=<0.05 ; b=<0.005 ; c=<0.0005 ;$ Effect size $($ Cohen's $d)-{ }^{*}=0.2-0.4($ small);

${ }^{* *}=0.5-0.8$ (medium); ${ }^{* * *}=>0.8$ (strong);

$(+)=$ improvement, $(-)=$ deterioration;

Facilities comparison $P$ value $-0=$ not significant; $1=<0.05 ; 2=<0.005 ; 3=<0.0005$.

the intervention; however significant $(\mathrm{p}<0.0005 ; \mathrm{d}=4.27)$ improvement was observed after the intervention. Previous studies reported 67\% [23] and $72-75 \%[8]$.

More than $60 \%$ of the parents could not measure $5 \mathrm{ml}$ correctly; about $47 \%$ overdosed while the remaining under-dosed. The consequences of a single dosing error may be minimal. But these types of overdosing and under-dosing errors are likely to accumulate especially in children that were administered $\operatorname{drug}(\mathrm{s})$ every four to eight hours for several days and accounted for some of the $\mathrm{C}$ and $\mathrm{D}$ errors recorded in this study. Other causes of $\mathrm{C}$ and $\mathrm{D}$ errors observed were the inability of parents to state the administration time precisely i.e. every 6,8 or 12 hours for qds, tid and bd) and the number of days the drugs should be given accurately. Most parents $(>60 \%)$ would have given the drugs within 12 hours irrespective of the frequency of dosing interval ( 8 am to $8 \mathrm{pm}$ ); this could lead to development of resistance and treatment failures. Look-alike, anxiety and inadequate information from the health care providers could be the cause of the errors recorded. With proper training, parents can dose liquid medication accurately, understand and adhere to correct dosing timing and duration as evidenced in the result obtained after the intervention.

\section{Conclusion}

This study provides insights into the drug administration practice in paediatric outpatient departments in Kano, Nigeria. Though drug administration errors were extremely frequent in this study, and many had more than one error, the intervention resulted to significant decrease the possibilities of drug administration error.

\section{References}

1. American Society of Hospital Pharmacists (1982) ASHP Standard definition of a medication error. Am J Hosp Pharm 39: 321

2. Avenel S, Bomkratz A, Dassieu G, Janaud JC, Danan C (2000) The incidence of prescriptions without marketing product license in a neonatal intensive care unit. Arch Pediatr 7: 143-147.

3. Roberts R, Rodriguez W, Murphy D, Crescenzi T (2003) Pediatric drug labeling: improving the safety and efficacy of pediatric therapies. JAMA 290: 905-911.

4. Rowe C, Koren T, Koren G (1998) Errors by paediatric residents in calculating drug doses. Arch Dis Child 79: 56-58.

5. McIntyre J, Conroy S, Avery A, Corns H, Choonara I (2000) Unlicensed and off label prescribing of drugs in general practice. Arch Dis Child 83: 498-501.

6. Frey B, Buettiker V, Hug MI, Waldvogel K, Gessler P,et al. (2002) Does critica 
Citation: Chedi BAZ, Abdu-Aguye I, Kwanashie HO (2014) Evaluating Educational Intervention to Improve Drug Administration Practice in Out-Patient Children in Northwestern Nigeria. Adv Pharmacoepidemiol Drug Saf 3: 162. doi:10.4172/2167-1052.1000162

Page 4 of 4

incident reporting contribute to medication error prevention? Eur J Pediatr 161: 594-599.

7. King WJ, Paice N, Rangrej J, Forestell GJ, Swartz R (2003) The effect of computerized physician order entry on medication errors and adverse drug events in pediatric inpatients. Pediatrics 112: 506-509.

8. Miller MR, Robinson KA, Lubomski LH, Rinke ML, Pronovost PJ (2007) Medication errors in paediatric care: a systematic review of epidemiology and an evaluation of evidence supporting reduction strategy recommendations. Qual Saf Health Care 16: 116-126.

9. Nsimba SE (2006) Assessing prescribing and patient care indicators for children under five years old with malaria and other disease conditions in public primary health care facilities. Southeast Asian J Trop Med Public Health 37: 206-214.

10. Hyam E, Brawer M, Herman J, Zvieli S (1989) What's in a teaspoon? Underdosing with acetaminophen in family practice. Fam Pract 6: 221-223.

11. Kohn LT, Corrigan JM and Donaldson MS (2000) Committee on quality of health care in America. In: To Err Is Human: Building a Safer Health System. Washington, DC: National Academy Press, 1-287.

12. Kaushal R, Jaggi T, Shojania KG, Bates DW and Walsh K (2004) Pediatric medication errors: what do we know? What gaps remain? Ambulatory Pediatrics 4:73-81.

13. Grasso BC, Rothschild JM and Genest R (2003) What do we know about medication errors in inpatient psychiatry? Joint Commission Journal on Quality and Safety, 29:391-400.

14. http://www.nccmerp.org/pdf/taxo2001-07-31.pdf
15. Tumwikirize WA, Ekwaru JP, Mohammed K, Ogwal-Okeng JW and Aupont O (2004) Proceeding of the International Conference on Improving Use of Medicines. $2^{\text {nd }}$ International Conference on improving Use of Medicines, Chiang Mia, Thailand.

16. Dimitrov MD and Rumrill PD (2003) Pretest-posttest designs and measurement of change. WORK: A Journal of Prevention, Assessment, \& Rehabilitation 20 159-165.

17. Abiola OO (2007) Procedures in Educational Research: Hanijam Publications Kaduna, Nigeria.

18. Cohen J (1998) Statistical power analysis for the behavioral sciences. (2ndedn), Hillsdale, NJ, United States.

19. Guyon AB, Barman A, Ahmed JU, Ahmed AU and Alam MS (1994) A baseline survey on use of drugs at the primary health care level in Bangladesh. Bull World Health 72: 265-271.

20. Krause G, Borchert M, Benzler J, Heinmuller R, Kaba I, et al. (1999) Rationality of drug prescriptions in rural health centers in Burkina Faso. Health Policy Plan, 14: $291-298$

21. Chareonkul C Khun VL and Boonshuyar C (2002) Rational drug use in Cambodia: study of three pilot health centers in Kampong Thom Province. Southeast Asian J Trop Med Public Health 33: 418-424.

22. Rishi RK, Sangeeta S, Surendra K and Tailang M (2003) Prescription audit: experience in Garhwal (Uttaranchal), India. Tropical Doctor, 33: 76-79.

23. McMahon SR, Rimsza ME and Bay RC (1997) Parents can dose liquid medications accurately. Pediatrics 100:330-333. 